\title{
Pharmacosomes and Emulsomes: An Emerging Novel Vesicular Drug Delivery System
}

\author{
Uzma Afreen and Krishna Shailaja $A^{*}$ \\ Department of Pharmaceutics, RBVRR Women's College of Pharmacy, Affiliated to Osmania University, Barkatpura, India
}

*Corresponding author: Krishna Shailaja A, Department of Pharmaceutics, RBVRR Women's College of Pharmacy, Affiliated to Osmania University, Barkatpura, Hyderabad Osmania University-500027, India

\begin{abstract}
Novel drug delivery attempts to either sustain drug action at a predetermined rate, or by maintaining a relatively constant, effective drug level in the body with concomitant reduction of undesirable side effects. In vesicular drug delivery system drug binds covalently to the lipid molecule by which the drug release in a controlled manner and also drugs which are of hydrophilic or lipophilic nature can be delivered by using the vesicular drug delivery systems. The release of drug from the vesicles depends on the physicochemical characteristic of both the drug and carrier. Vesicular drug delivery system contains liposomes, niososmes, transferosomes, pharmacosomes, emulsomes, electrosomes, ethosomes etc. Pharmacosomes are amphiphilic phospholipids complexes of drugs bearing active hydrogen that bind to phospholipids. Like further vesicular systems pharmacosomes provide an effective method for delivery of drug directly to the site of infection, leading to minimization of drug toxicity with no adverse effects. They also decrease the cost of therapy by enhancing bioavailability of medication, particularly in case of poorly soluble drugs. Emulsomes is a novel lipoidal vesicular system with an inner solid fat core surrounded by a phospholipid bilayer. Emulsomal formulations composed of solid lipid core material and stimulated by cholesterol and soya lecithin. The drug is loaded accompanied by sonication to produce emulsomes of small size. This review discourse the concept of emulsome and pharmacosome drug delivery system, summarize the success of emulsomes and pharmacosomes for the delivery of small molecules, and special concentration has been paid to its formulation design, merits, biopharmaceutical aspects, stability aspects, and various different aspects associated to the drug delivery system including future aspects. Hence, pharmacosomes and emulsomes opens new challenges and opportunities for improved novel vesicular drug delivery system.
\end{abstract}

Keywords: Controlled oral drug delivery; vesicular drug delivery; emulsomes; pharmacosomes; oral bioavailability

\section{Introduction}

In the past few decades, significant attention has been made on the development of novel drug delivery system (NDDS). The NDDS should ideally fulfil two conditions: Firstly, it should deliver the drug at a rate directed by the requirement of the body, over the period of treatment, secondly; it should channel the active system to the site of action. Conventional dosage forms as well as prolonged release dosage forms are unable to meet none of these. Novel drug delivery efforts to either sustain drug activity at a predetermined rate, or by maintaining a relatively constant, effective drug level in the body with concomitant reduction of undesirable side effects. It can also localize drug action by spatial positioning of controlled release systems adjacent to, or in the diseased tissue or organ; or target drug action by using carriers or chemical derivatization to deliver drug to specific target cell type [1]. Currently, vesicles as a carrier system have become the vesicles of option in drug delivery and lipid vesicles are accepted to be valuable in immunology, membrane biology, and diagnostic technique, and most importantly in genetic engineering [2,3] Lipids are mentioned to a group of naturally occurring molecules which contains fats, waxes, sterols, fat-soluble vitamins (such as vitamins A, D, E, and K), as well as mono-glycerides, triglycerides, phospholipids. Lipids may be widely defined as hydrophobic or amphiphilic small molecules; the amphiphilic nature of some lipids permits them to form structures such as that of vesicles [4-10]. Therefore, researchers realize the potential of the lipids for delivering soluble materials for prolong periods. Vesicles are unilamellar or multilamellar 
spheroid structures consist of lipid molecules assembled into bilayers, Because of their capacity to carry a number of drugs, vesicular delivery systems have been widely investigated for their prospective application in pharmaceutics such as drug delivery for drug targeting, for controlled release, or for increasing solubility $[5,10]$.

\section{Pharmacosomes}

Pharmacosomes are portion of the novel drug delivery system. They were first introduced by vaizoglu and Speriser in 1968. Pharmacosomes are determined as the colloidal dispersions, drugs covalently bound to the lipids, and may exists as ultra-fine vesicular, micellar, or hexagonal aggregates, on the basis of the chemical structure of the drug-lipid complex. The system is composed by linking a drug (pharmakon) to a carrier (soma), so they termed as "Pharmacosomes" [6,5]. After absorption, their velocity of deterioration into active drug molecule depends to a great extent on the size and functional groups of drug molecule, the chain length of the lipids and the spacer [8,3] (Figure 1).

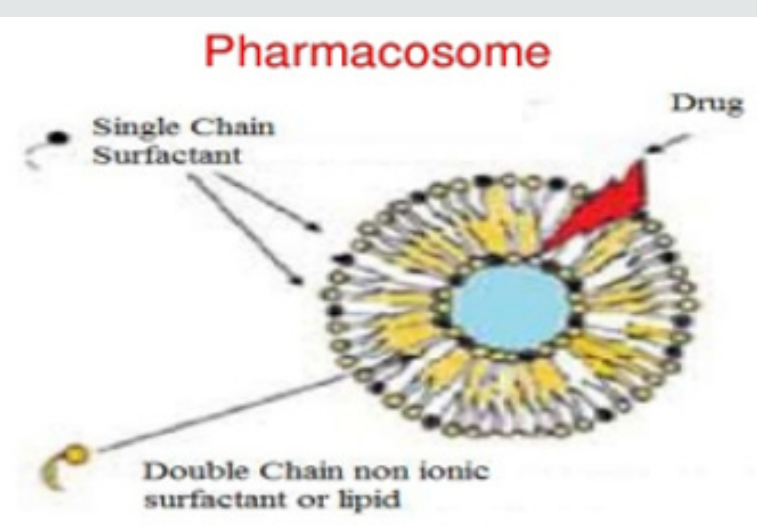

Figure 1: Pharmacosome.

a. Pharmacosomes assist in drug targeting and controlled release of drug to achieve desired dose.

b. They bearing unique advantage over liposome and niosome vesicles and are serve as an altering to conventional vesicles.

c. Problems like drug incorporation, leakage, or insufficient shelf life of drug can be avoided in pharmacosomes.

d. The concept of creation of the vesicular pharmacosomes is in accordance with bulk interaction and surface of lipids with drug substances.

e. Drugs occupying an active hydrogen atom $(-\mathrm{COOH},-\mathrm{OH}$, $-\mathrm{NH}_{2}$, etc.) can be esterified into the lipid form, with or without spacer chain that results in formation of an amphiphilic compounds, which helps in the permeation into the target site.

f. The prodrug amalgamates have both hydrophilic and lipophilic properties, so they acquire amphiphilic nature and therefore found to decrease the interfacial tension, and mesomorphic behavior at elevated concentrations $[7,9]$.

\section{Advantages of Pharmacosomes $[3,7,10]$}

Compared to other categories of lipid based delivery systems, pharmacosomes exhibit better results in many ways.

a. The drug-lipid complex depends upon the phase transition temperature but independent on rate of release as it is covalently bounded to the lipid.

b. No leaching will occur as drug is bounded to the lipid by covalent bonding.

c. No problem is associated with drug incorporation.

d. The physicochemical stability of the pharmacosomes depends on the physicochemical properties of the drug- lipid complex.

e. The metabolism of the drug depends on the spacer, length of chain in lipid, functional groups and size of the drug during its absorption.

f. They are suitable for the both lipophilic and hydrophilic drugs.

g. The aqueous solution of the amphiphiles exhibits concentration dependant aggregation.

h. The drug and carrier are covalently linked together so, entrapment efficiency is high and predetermined.

i. Delivers drug at the specific site and site targeted.

j. They improves the bioavailability majorly incase of poorly soluble drugs.

k. They reduce the adverse effects and toxicity.

l. In pharmacosomes, there is no require to remove the unentrapped drug when compared to liposomes where the free drug should be removed.

m. There is reduction cost of therapy.

\section{Disadvantages of Pharmacosomes $[7,11]$}

- Water insoluble drugs are encapsulated relatively in a less hydrophobic region within membrane bilayer rather than relatively large surface area.

- Synthesis of a compound depends on its amphiphilic nature.

- $\quad$ Preffered surface and bulk interaction of lipids with drugs.

- $\quad$ Required covalent bonding to protect the leakage of drugs.

- The storage of pharmacosomes undergoes fusion and aggregation and also chemical hydrolysis. 


\section{Limitations of Pharmacosomes $[3,12]$}

$>$ A compound can be synthesised depending on the amphiphilicnature.

> They require superficial as well as mass drug-lipid interaction.

$>$ Covalent type of bond is required to restrict drug leakage.

$>$ Pharmacosomes are susceptible to get fused, aggregate,orhydrolysebychemicalsonstorage.

\section{Salient Features of Pharmacosomes [7]}

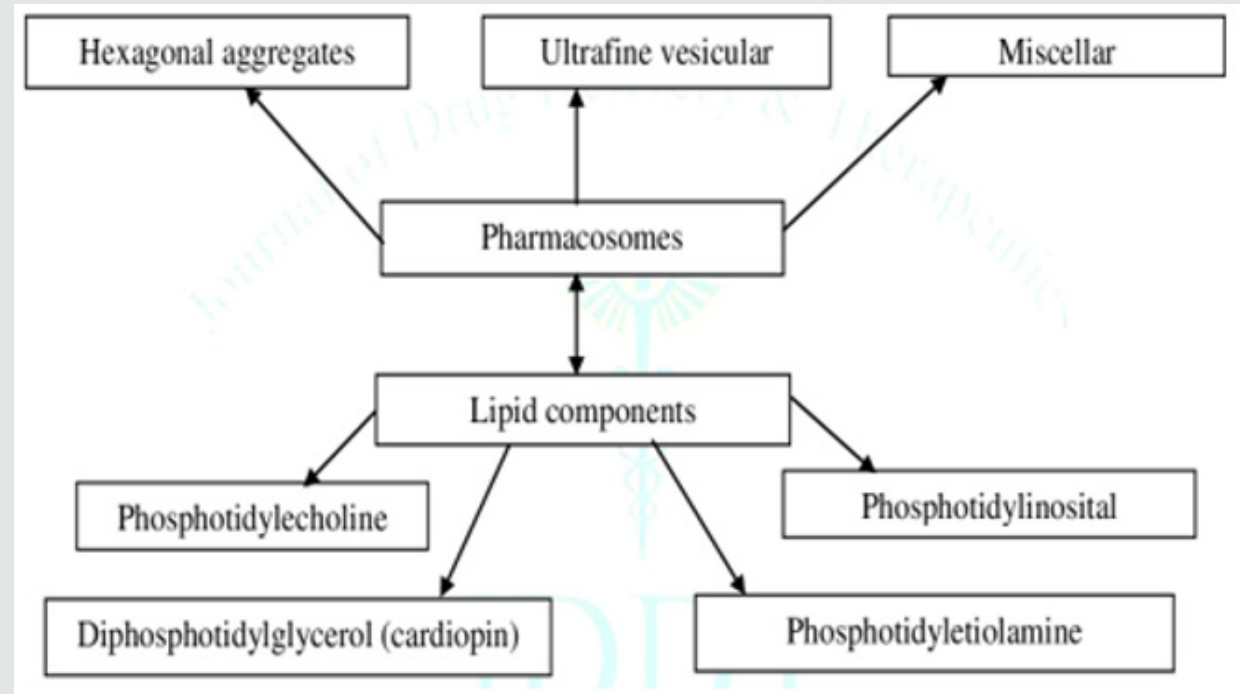

Figure 2: Components of pharmacosomes.

o Pharmacosomes can be administered in various route such as orally, extra vascular and intravascular.

o The drug conjugates with lipids and results in high entrapment efficacy in predetermined fashion.

o There is no problem when the drug is incorporated into the lipid.

o They consist of both hydrophilic and lipophilic properties so they can easily pass through the cell membrane, walls, or tissues either by action of endocytosis or exocytosis.

o The physicochemical characters of the drug-lipid complex affect the stability of pharmacosomes. (Figure 2).

\section{Components of Pharmacosomes}

1. Drugs: Any drug containing an active hydrogen atom (- $\mathrm{COOH}, \mathrm{OH},-\mathrm{NH}_{2}$ etc) can be esterified to the lipid, with or without spacer chain resulting into amphiphilic complexes. Facilitates membrane, tissue, cell wall transfer in the organisms is due to its amphiphilic nature $[3,13]$

2. Solvent: Organic solvent of analytical grade and intermediate polarity is used in development of pharmacosomes. It must be of high purity and volatile in nature. The phospholipids and the drug must be dissolved in the selected solvent. The selection of solvent depends on polarity of the drug and the lipid $[3,10]$

\section{Lipids:}

- $\quad$ Phospholipids are the major components of biological membrane; majorly two types of phospholipids are used namely phosphoglycerides and sphingolipids.

- The most common kind of phospholipids is Phosphotidylecholine moiety.

- Phosphotidylecholine is an amphiphilic molecule in which a glycerol bridges joints a pair of hydrophobic acylhydrocarbon chains with hydrophilic polar head group phosphocholine [7].

\section{Preparation of Pharmacosomes}

\section{Hand Shaking Method/Solvent Evaporation Method}

Mixture of drug and lipid is dissolved in a volatile organic solvent. Thereafter solvent is evaporated using rotatory evaporator in round bottom flask which leaves a thin film of solid mixture deposited on the walls of flask. The dried film hydrates with aqueous medium and readily gives a vesicular suspension $[3,14]$.

\section{Ether Injection Method}

The drug-lipid complex is dissolved in specified volume of ether. Then the above mixture is slowly injected into a heated buffer solution, resulting in the formation of the vesicles. The nature of vesicle especially the shape depends on the concentration. The 
variety of structures may be formed that are, round, cylindrical, disc, cubic, or hexagonal type depending on the amphiphilic state $[7,15]$.

\section{Anhydrous Co-Solvent Lyophilization Method}

First of all drug and phospholipids are dissolved in solution of dimethyl sulfoxide containing glacial acetic acid. Then mixture is agitated to get clear liquid and then freeze-dried overnight at condenser temperature. The resultant complex is flushed with nitrogen and stored at $4^{\circ} \mathrm{C}[3,16]$.

\section{Supercritical Fluid Process}

Drug and lipid complex are dissolved in a supercritical fluid of $\mathrm{CO}_{2}$, then it is mix into nozzle mixing chamber $[3,7]$.

\section{Evaluation of Pharmacosomes}

i. Complex determination: Using correlation spectrum the formation of both conjugate and complex can be contingent upon inspecting with that of discrete constituents and with their mixture using FTIR spectrum [17].

ii. Surface morphology: The surface morphology can be predicted using Scanning Electron Microscopy (SEM) or Transmission Electron Microscopy (TEM). The shape and size of pharmacosomes are prone to variations by some variables such as rotation speed, vaccum applied, Purity grade of phospholipids or the method used [7].

iii. Drug content:To determine the drug content, the amount of drug present in the drug complex estimated quantity of pharmacosomes were transferred into a suitable flask containing known volume of solvent in which drug is soluble. The solution is mixed by using magnetic stirrer. After 24 hrs suilatble dilution of drug content is determined by UV spectrophotometrically [18,3].

iv. Solubility: Solubility is determined by placing the known amount of phospholipid complex in a screw caped penicillin bottle containing aqueous phase buffer solution of varying $\mathrm{pH}$ (27.4) and organic phase like 1- Octonol with continuous shaking at a temperature of $37^{\circ} \mathrm{C}$ for $24 \mathrm{hrs}$. Then both the layers will be separated and samples were analyzed using HPLC or UV spectrophotometer [7,18].

v. Differential scanning calorimetry (DSC): This thermal analytical technique is used to determine the drug-excipients compatibility or interactions. The interaction can be concluded by the elimination endothermic peaks, appearance of peaks and change in peak shape and its onset, peak temperature/melting point and relative peaks area or enthalpy [3]

vi. X-ray power diffraction (XRPD): It is performed to detremine the degree of crystallinity by using the relative integrated intensity of reflection peaks . The itegrated intensity is specified by the area under curves of the XRPD patterns and it represents the specimen charetristics [19,3].

vii. Fourier transform infrared spectroscopy (FTIR): With the help of IR spectroscopy the formation of complex can be confirmed by comparing the spetrum of complex with the spectrum of individual components and their mechanical mixture [3].

viii. Dissolution studies: Dissolution studies in vitro are done by using various models available using different buffers, then the results obtained are estimated on the basis of activity of the drug $[7,20]$.

ix. In vitro drug release rate: The in vitro drug release rate is estimated by reverse dialysis bag technique. In this method pharmacosomes are inject inside the dialysis bag and the receiver phase is placed outside. Dialysis bag containing the continuous phase and they are suspended in a vessel containing the donor phase and stirred at predetermined time intervals, each dialysis bag is removed and the contents are analyzed for drug release. An advantage of this technique is increase in the membrane surface area available for transport from the donor to receptor compartment. Another advantage of this technique is the increased efficiency in terms of staffing as a consequence of reduction in number of steps $[7,21]$.

\section{Applications of Pharmacosomes [3,7]}

- Pharmacosomes possess better stability and shelf life compared to other vesicular drug delivery systems.

- Absorption and permeation of the drug can be enhanced formulating in to pharmacosomes.

- The transportation of drug across the biological membranes is due to the vesicular formation as they have the capacity to interact with the membranes, due to their transitions from vesicle to micelle by altering the transition temperature.

- By altering the temperature at the targeting site pharmacosomes have the capacity to deliver the drug in the targeted site especially in the case of cell specific drug vehicles.

- Pindolol diglyceride, Amoxicillin, Taxol, Cytrabine, Dermatansulfate, Bupranolol hydrochloride etc showed increase in pharmacological action by formulating into pharmacosomes.

- The mechanism of action of drugs and non bilayer phases can be studied by using pharmacosomes.

- $\quad$ PEGylation and biotinyzation are used in current research in the production of pharmacosomes.

- Ophthalmic drug delivery with a modified corneal drug 
transport and release by diluting with tears where the drug should be of amphiphilic in nature.

- Phytoconstituents such as flavonoids, glycosides, xanthones etc, shows both increase in pharmacokinetic and pharmacodynamic actions.

- The ability of transportation of biological components like proteins and amino acids by using pharmacosomes.

- Pharmacosomes employed to prepare Tetrahydrofuran injection method and studied in the in-vitro behavior in rats for didanosine, by which they observed that pharmacosomes possess prolonged action in both targeted site and liver.

- Semalty and colleagues, studied the developmentofpharmacosomesofaceclofenacandevaluated them. A higher drug content was found to be $91.88 \%(\mathrm{w} / \mathrm{w})$ for 1:1 aceclofenac phospholipid complex and $89.03 \%(w / w)$ for 2:1 aceclofenac phospholipid complex. aceclofenac pharmacosomes showing highest solubility than aceclofenac. Moreover,the drug release over $4 \mathrm{hrs}$ of dissolution study was only $68.69 \%$ in case of free aceclofenac, while it was found to be $79.78 \%$ for $1: 1$ aceclofenac pharmacosome and $76.17 \%$ for $2: 1$ aceclofenac pharmacosomes for the same time span.

- Semalty et al., studied the development of diclofenac pharmacosome, and it was found that solubility was increased in pharmacosomes $(22.1 \mu \mathrm{g} / \mathrm{mL})$ as compared to diclofenac $(10.5 \mu \mathrm{g} / \mathrm{mL})$.Drug release was also enhanced from $60.4 \%$ to $87.8 \%$ of diclofenac pharmacosomes after $10 \mathrm{hrs}$ of dissolution study. Observed drug content of diclofenac pharmacosomes was found to be $96.2 \pm 1 \%$.

- Han and colleagues, optimized the preparation of 20(S)-protopanaxadiol pharmacosomes and observed the encapsulation efficiency of pharmacosome, which was found to be $80.84 \pm 0.53$ for a diameter of $100.1 \mathrm{nmand} 72.76 \pm 0.63$ for $117.3 \mathrm{~nm}$ diameter.

- Pingetal et al., has worked ondidanosinepharmacosomes using tetrahydrofuran injection method and studied the in vivo behaviour in rats. It was observed that pharmacosomes may be a potential delivery system for prolonged effects in targeted tissues and liver targeting.

- Zhangetal et al, usingcentralcompositedesign,regulated pharmacosomes of 3,5-dioctanoyl-5-fluoro2-deoxyuridine and observed excellent targeting efficiency of pharmacosomes invivo and enhanced drug potential to pass through blood brain barrier.

- Yi-Guang et al., has worked to prepare acyclovir pharmacosomes and observed that the plasma proteins in bloodabsorbedpharmacosomesandinterferedwith the interactions of erythrocytes and hence minimize haemolyticreaction.

- Semalty et al., prepared aspirin-phospholipid complex (1:1molar ratio) and observed the enhanced bioavailability of aspirin and reduced gastrointestinal toxicity.

\section{Emulsomes}

Emulsome is a lipid based drug delivery system, mainly designed for parenteral delivery of drugs possess poor aqueous solubility [22,23] Nanosize Lipid particles (bio adhesives nano emulsion) consisted of microscopic lipid assembly with a polar core in emulsomes. The internal core is made up of fats and triglycerides, which are stabilize in form of $\mathrm{o} / \mathrm{w}$ emulsion by addition of high concentration of lecithin. Emulsomes have the characteristics of both liposomes aa well as emulsions. By asset of solidified or semi solidified interior oily core, it provides better opportunity to load lipophilic drugs in high concentration, simultaneously a controlled release can also be expected and these also have the ability to encapsulate water soluble medicaments in aqueous compartments of surrounding phospholipids layers. The solvent-free and surfactant-free emulsomes technologies have demonstrated high encapsulation capacity for water insoluble antifungal and anticancer drugs, showed enhanced drug delivery and improved preclinical efficacy for oral route [23,24]. This study focuses on preparing macrophage (liver, spleen and bone marrow) targeted Emulsomes to reduce the adverse effects of conventional treatments [23,25]. (Figure 3)

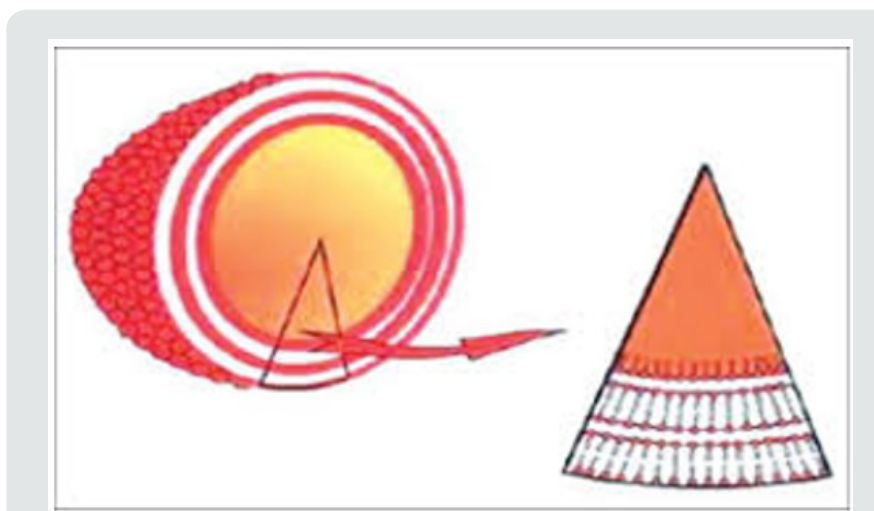

Figure 3: Structure of emulsome.

\section{Advantages of Emulsomes [26,27]}

- Emulsomes protect drug from harsh gastric environment of stomach before oral administration because the drug is surroundedby the triglyceride lipid core. This hypothesis could be supported by the fact that gastric $\mathrm{pH}$ and gastric enzymes are unable to hydrolyze triglycerides.

- Emulsomes enhance the solubility and bioavailability of poorly aqueous soluble drugs. They are composed of triglycerides and form micelles or are organized as lipid bilayers with the hydrophobic tails lined up against one another and the 
hydrophilic head-group facing the water on both sides. These unique features make phospholipids most suitable to be used as excipients for poorly water soluble drugs.

- $\quad$ Emulsomes are composed of lipid core. Lipids are used to develop oral controlled delivery of drug. The wide spread adoption of lipid based strategies for enhancing drug exposure is limited.

- Emulsomes are economical alternative to current commercial lipid formulations because they minimize the dosing frequency of drug.

- Emulsomes-based system showed excellent potential for targeting. The formulations could significantly modify providing prolonged action at comparatively low drug doses thereby reduction in the toxicity problem due to complimentary localization of the drug in target cells.

- $\quad$ They provide significantly modify the pharmacokinetics of drugs. Also, they resist development of multi drug resistance, often associated with over expression of a cell membrane glycoprotein, which cause efflux of the drug from the cytoplasm and results in an incapable drug concentration inside the cellular compartment.

\section{Disadvantages of Emulsomes}

- $\quad$ Emulsomes are particularly helpful for administration of poorly water-soluble lipophilic drugs which heretofore either could not be administered parenterally or, if so administered, would cause unacceptable side-effects.

- One major disadvantage of standard oil-in-water emulsions is minimal drug loading. When drug encapsulation above $1 \%$ is needed, a correspondingly larger oil phase (10$20 \%$ ) is required to dissolve the drug. Although, the high oil content decreases the stability of the emulsion, and the addition of a surfactant or cosurfactants, is mandatory.

- Due to the detergent properties of most surfactant compounds, their use for parenteral administration is very limited.

- Many toxic reactions have been reported even with the surfactants already approved for intravenous formulations, as in the case of Fungizone containing sodium deoxycholate.

\section{Mechanism of Emulsomes Absorption}

Emulsomes have structural similarity with the chylomicrons (natural lipoprotein of the body) and therefore expected to mimic these lipoproteins in behavior. These diminutive lipids like particles are frequently taken through endogenous lipid absorption mechanism through enterocytes of GIT tract [28]. Intestinal absorption of long-chain triglyceride from enterocytes is a complex incident that includes the co-ordination of synthesis of apolipoproteins and lipids and their intracellular assembly into mature lipid-containing particles [26]. The major digestive products of triglyceride are monoglycerides and free fatty acids. These are absorbed into the enterocytes via passive diffusion and transported within the enterocyte to the endoplasmic reticulum (ER), where biosynthesis of complex lipids to form triglyceride takes place. The precursors of chylomicrons, termed prechylomicrons, are synthesized in the ER and Golgi apparatus. Lipoproteins are made in the ER and then transported to the Golgi. The chylomicrons then migrate to the lateral membrane of enterocytes and are exocytosed, and the triglyceride-rich lipoproteins are discharged into the intercellular space. Lipid-based excipients can influence oral absorption via different physiological effects such as retarded gastric emptying, stimulating bile flow, and secretion of pancreatic juice increasing the membrane lipid fluidity or acting straight onto enterocytes-based drug transport and disposition [26,27].

\section{Emulsomal Formulation}

a. Lipid core: An important component of emulsomes is an internal hydrophobic core or lipid core includes lipid, which exhibits solid or lipid crystal phase or mixed solid and liquid crystal phase at room temperature $\left(25^{\circ} \mathrm{C}\right)$. There are abundant lipids or lipid like excipients available commercially. All of which are collectively called lipids in the pharmaceutical field.The lipid used may be single or mixture of lipids. They are fatty acids and their derivatives or substances biosynthetically and functionally related to these compounds. Lipids are generally insoluble in water and are often identified by their fatty acid composition, melting point, and hydrophiliclipophilic balance (HLB). Lipids with low HLB and high melting point are suitable for sustained release. Whereas, the semi-solid excipients and those with high HLB serve as immediate release and bioavailability enhancement excipients. Triglycerides which are solid at $25^{\circ} \mathrm{C}$ are found to be appropriate core material because these lessen the acceptable storage life of o/w emulsion. The triglycerides which are used for preparation of emulsome composed of un-branched fatty acid with chain length in the C-10 to $\mathrm{C}-18$ range [26-30].

b. Antioxidant: The lipid core of emulsome particles of this design optionally may contain one or more antioxidant. The preferred antioxidant is a-tocopherol or its derivative, which are members of vitamin E family. Other antioxidants include butylated hydroxytouline (BHT). Antioxidants lessen the formation of oxidative degradation products of unsaturated lipids such as peroxides. The need of antioxidant may be protected by preparing the lipid core form saturated fatty acid [26,31].

c. Negatively charged particles: Negatively charged lipid particles like oleic acid or negatively charged phospholipids like phosphatidic acid, phosphatidylinositol, and phosphatidylsterine can be imparted to emulsomes to increase the zeta potential of the composition, thus stabilizing the particles. Additionally, the 
incorporation of these negatively charged lipids compounds in emulsomes result in the development of phospholipids bilayers with opposing charge. Thus, enhancing the loading aqueous compartment formed by the phospholipids bilayers surround the lipid core. This effect results from the layers aqueous space between the bilayers cause electrostatic repulsion between them. Negative charge reduces the particles aggregation, which minimizes the coalescence, floccation, or fusion [27, 32].

d. Surfactants: Selection of surfactant should be done on the basis of Hydrophilic Lipophilic Balance (HLB) value. As HLB is a good indicator of the vesicle forming ability of any surfactant, HLB number in between 4 and 8 was found to be compatible with vesicle formation.Transition temperature of surfactants also influence the entrapment of drug in vesicles. Spans with high phase transition temperature provide the high entrapment for the drug and vice versa. The drug leaching from the vesicles is decreased due to high phase transition temperature and low permeability. High HLB value of Span 40 and 60 results in reduction in surface free energy, which allows forming vesicles of larger size and hence large area exposed to the dissolution medium $[26,27]$.

e. Phosphatidyl choline: Phosphatidyl choline is a major component of lecithin. Phosphatidyl choline has low solubility in water. In aqueous solution its phospholipids can form bilayer sheets, micelles, or lamellar structures, depending on hydration and temperature. This results in a type of surfactant that is usually classified as amphipathic. They are a major component of biological membranes and can be easily obtained from a variety of readily available sources such as egg yolk or soya beans. Depending upon the source from which they are acquired they are named as egg lecithin and soya lecithin. Incorporation of lecithin further enhanced the percent drug entrapment to $96.1 \%$ and leads to vesicles of smaller size due to increase in hydrophobicity, which results in depletion of vesicle size [33].

f. Cholesterol: Cholesterol is an essential component of emulsomes as vesicles. Incorporation of cholesterol effect the vesicles stability. Concentration of cholesterol plays a crucial role in entrapment of drug in vesicles. There are describes that entrapment efficiency increase with increasing cholesterol content. It was observed that with very high cholesterol content had a lowering effect on drug entrapment to the vesicles. This could be due to the fact that cholesterol beyond a certain level starts disturbing the regular bilayer structure leading to loss of drug entrapment [26]. Table 1 (Figure 4)

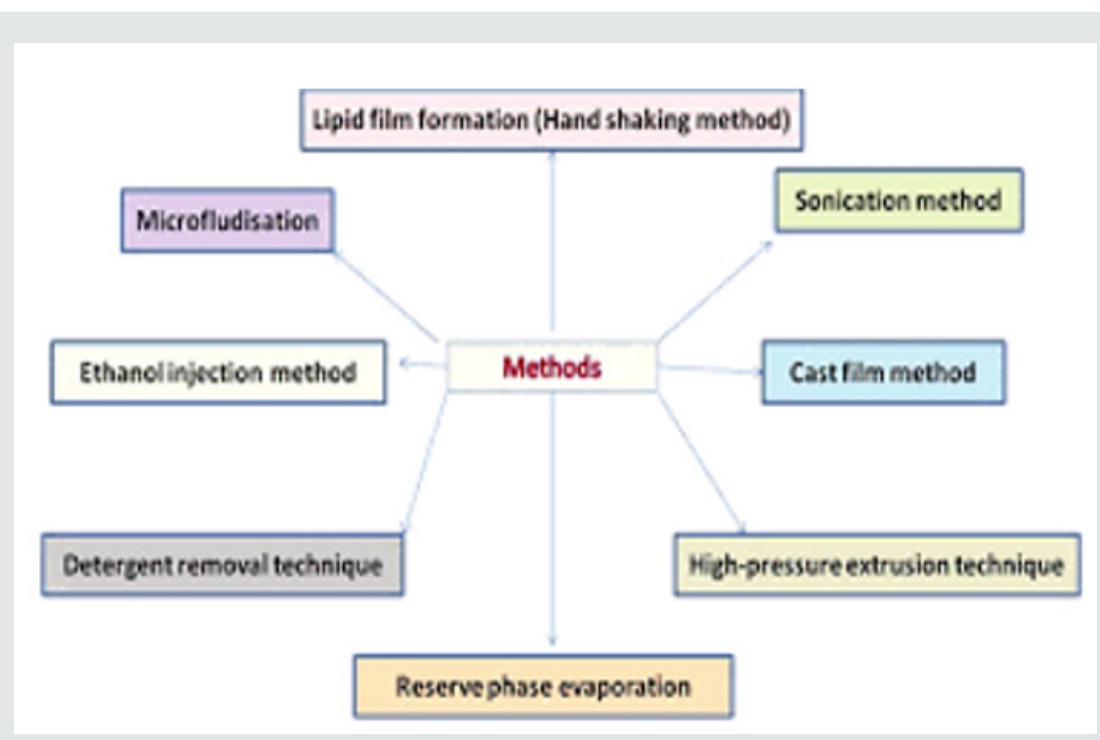

Figure 3: Methods of Emulsomal formulation.

Table 1: Various materials used for emulsomes formulation.

\begin{tabular}{|c|c|c|}
\hline Materials & Uses & References \\
\hline Triglycerides & Used as hydrophobic lipid core, Lipids with low HLB value provides sustain released formulation. & 35,36 \\
\hline Cholesterol & $\begin{array}{l}\text { Incorporation of cholesterol influence vesicles stability, excessive cholesterol leads to un-stability of } \\
\text { the formulation. }\end{array}$ & 37 \\
\hline Soya lecithin & Bilayer sheets, micelles or lamellar structures and also increases the entrapment efficiency. & 32 \\
\hline Sterylamine & Impart positive charge for target delivery and raised the zeta potential of formulation. & 37 \\
\hline Antioxidant & Protect the lipids from oxidation or rancidity. & 31 \\
\hline Surfactants & Provide the highest entrapment for the drug. & 38 \\
\hline Charged particles & Zeta potential of the composition, stabilizing the particles and reduce the particles aggregation. & 28,36 \\
\hline
\end{tabular}




\section{Methods of Preparation [26,27]}

Lipid film formation (Handshaking method): In this method surfactants/lipids are casted as layers of film form their organic solution using flask rotary evaporator under reduce pressure (or by hand shaking) and then casted films are dispersed in aqueous medium.Upon hydration, the lipids swell and peel off from the wall of round bottom flask at temperature slightly above the phase transition temperature of surfactants used for specific period of time (time of hydration) with contineous mild shaking. The mechanical energy is required for the swelling of lipid and dispersion of casted lipid film is imparted by manual by manual hand shaking or by exposing the film to a steam of water saturated nitrogen for 15 minutes followed by swelling in the aqueous medium without shaking. Multi lamellar vesicles (MLV) are produced by hand shaking method while large unilamellar vesicles (LUVs) are produced by non shaking method.

Reserve phase evaporation: This technique, so-called 'reverse-phase evaporation' or REV method is comprised of two steps. Prepare a w/o emulsion of phospholipids and buffer in excess organic phase. Remove organic phase under reduced pressure. The two phases (phospholipids and water) are usually emulsified by mechanical methods or by sonication. Removal of the organic solvent under vacuum causes the phospholipid coated water droplets to come together to form a gel-like matrix. Further removal of organic solvent, under reduced pressure, causes the gel-like matrix to form into a paste of smooth consistency. This paste is a suspension of LUV. Drug entrapment efficiencies up to $60-65 \%$ can be achieved by this method. This method was used to encapsulate both small and large molecules. The main disadvantage of this method is the exposure of drug to be encapsulated to organic solvents and to mechanical agitation. In this procedure, phospholipids are dissolved in organic solvents such as chloroform, isopropylether, or freon. In order to promote conditions for good emulsification, it may sometimes be required to mix two organic solvents to adjust the density to unity that is closer to the density of aqueous phase. Biologically active molecules such as enzymes, protein pharmaceuticals, and RNA type molecules may undergo conformational changes, protein denaturation, or breakage of DNA strands due to the harsh conditions of organic solvent exposure and mechanical agitation.

High-pressure extrusion technique: It was demonstrated by several researchers that when MLV are repeatedly passed through very small pore polycarbonate membranes ( 0.8 to $1.0 \mathrm{pm})$ under high pressure the average diameter of the vesicles become progressively smaller reaching a minimum of 60-80 nm after 5-10 passes. As the average size is reduced the vesicles tend become unilamellar. Similar results are noted by other researchers when MLV were passed through a Microfluidizer. Microfluidizer is an instrument that forces the feed material under high pressure by narrow orifice. It appears that when MLV are forced through the small orifice, layers of bilayers are removed from the vesicular structure, as if the layers of onion skin are separated when it is peeled. It was also suggested that the mechanism of layer separation is only applicable to vesicles made with positively charged phospholipids and the vesicles that are greater than $70 \mathrm{pm}$ in size.

Sonication method: Solid lipids, cholesterol, and phosphatidylcholine in different molar ratios were taken in a roundbottom flask and dissolved in a minimum quantity of chloroform containing 3 or 4 drops of methanol. To this solution, an accurately weighed quantity of drug was dissolved. The organic solvent was evaporated until complete dryness under reduced pressure using a rotary evaporator to form a thin lipid film on walls of theround bottom flask. The dried film was hydrated with phosphate-buffered saline $\mathrm{pH} 7.4(10 \mathrm{~mL})$ and homogenized by using ultrasonication for 15 minutes at $40 \%$ frequency to get emulsomes of nano-size range.

Cast film method: Emulsomes may be prepared by mixing phospholipids and triglycerides in a weight ratio range 0.5:1.0 where in the triglycerides has a solid to liquid phase transition temperature of greater than $25^{\circ} \mathrm{C}$. Suspend the mixture in an aqueous solution at a temperature below the solid to liquid transition temperature or else reduce the suspension to yield emulsomes. These emulsomes comprise a nanoemulsion of liquid particles having a mean particle diameter between 10-250 nm usually within the range 20 to 180 $\mathrm{nm}$ usually and frequency within range $50-150 \mathrm{~nm}$. The size range is ideally determined on a weight percentage basis rather than a particle number basis. Conventionally the lipid component may be volatile and chemically unreactive volatile organic solvent such as dichloromethane or diethylether. The solvent is removed typically under reduced pressure in a rotary evaporator or under stream of inert gas. The resulting lipid film is hydrated and dispersed by covering and shaking with an aqueous solution. If the drug component were not included in the organic solution, they may be added to aqueous hydration solution. The lipid suspension or dispersion is then sized typically by high shear homogenizer at a pressure up to 800 bars.

Ethanol injection method: This method has been describe as one of the alternatives used for the preparation of small unilammellar vesicles (SUVs). In this method, an ethanol solution of surfactant is injected quickly through a fine needle into excess of saline or other aqueous medium. Vaporization of ethanol leads to the formation of vesicles. In the ethanol injection method a narrow distribution of small liposomes (under $100 \mathrm{~nm}$ ) can be obtained by simply injecting an ethanolic lipid solution in water, i.e. in one step, without extrusion or sonication. The ethanol injection method is an appropriate technique to obtain the spontaneous formation of emulsomes having a small average radius. Alternately, the lipid or 
lipid mixture is dissolved in alcoholic solvent and an aliquot of 200, 500 , or $600 \mathrm{~mL}$ fast injected at room temperature, $1 \mathrm{~mL}$ syringe into the dispersant solution, which contains water or saline solution, of $9.8 \mathrm{~mL}$ further diluted to $1: 50,9.5 \mathrm{~mL}$ diluted to $1: 20$ or $9.8 \mathrm{~mL}$ diluted to $1: 17$, respectively. The solution was then vigorously handshaken for 20-30 seconds. Then the ethanol solution is fastinjected in a $5 \%$ glucose solution. The vesicles had shown average diameter of about $60 \mathrm{~nm}$ and may be stable for at least one week.

Detergent removal technique: In this method, phospholipids and a detergent are mixed together to form micellar mixtures. After that detergent removed from the preparation while the micelles progressively become richer in phospholipid content and finally the lipids come together to form single bilayer vesicles. Methods like dialysis, column chromatography, or adsorption onto bio beads can be used to remove the detergent from the preparation. The dialysis technique was first revealed for reconstituting biological membranes solubilized with detergents. This method is suitable for the preparation of emulsomes also. Detergents that are commonly used for this purpose are those that have high critical micelle concentration (CMC). Materials such as sodium cholate, sodium deoxycholate, and octylglycoside and further detergents of high CMC (in the order of 10-20 mM) are acceptable detergents for this work.In technique, detergent was removed by a flow through dialysis cell from phospholipid detergent mixture. It was disclosed that this technique yielded homogeneous population of single layered emulsomes with mean diameters of 50-100 nm.

\section{Applications of Emulsomes}

Drug targeting: One of the most helpful aspects of emulsomes is their capacity to target drugs. Emulsomes can be used to aim drugs to the reticulo-endothelial system. The reticuloendothelial system preferentially takes up Emulsomes. The intake of Emulsomal vesicles is managed by circulating serum factors called opsonins. These opsonins mark the vesicles for clearance. Such localization of drugs is used to treat tumors in animals known to metastasize to the liver and spleen. This fixing of drugs can also be used for treating parasitic infections of the liver. Emulsomes can also be used for targeting drugs to organs other than the reticuloendothelial system. A carrier system (such as antibodies) can be attached to vesicles totargetthemtospecificorgans $[38,26]$.

Anti-neoplastic treatment: Most antineoplastic drugs cause acute side effects. Emulsomes can alter the metabolism; prolong circulation and half life of the drug, thus minimizing the side effects of the drugs. Emulsomal entrapment of Methotrexate showed favourable effects over the unentrapped drugs, such as reduced rate of proliferation of the tumor and higher plasma levels accompanied by slower elimination $[26,39]$.

Leishmaniasis:Leishmaniasis is a disease in which a parasite of the genus Leishmania invades the cells of the liver and spleen.
Frequently prescribed drugs for the treatment are derivatives of antimony (antimonials), which in high concentrations can cause cardiac, liver, and kidney damage. Use of emulsome in tests conducted showed that it was possible to administer higher levels of the drug without triggering the side effects, and thus allowed greater efficacy in treatment $[26,40]$.

Use in Biotechnology: Emulsomes are used in studying immune response because of immunological selectivity, low toxicity, and greater stability. They are being used to study the nature of the immune response provoked by antigens. The in vitro permeation of estradiol from vesicular formulations through human stratum corneum was studied. The vesicles are composed of non-ionic n-alkyl polyoxyethylene ether surfactants. Two mechanisms are proposed to play an important role in vesicle-skin interactions, i.e., the penetration enhancing effect of surfactant molecules and the effect of the vesicular structures caused by their adsorption at the stratum corneum- suspension interface [26,27].

\section{Conclusion}

Because of the site specific targeting of drugs and plenty of other advantages, vesicular drug delivery system is acquiring popularity in present scenario. Drugs can be directly targeted to their site of action to inhibit toxic and undesired effects to other sites, further these can be used for bioavailability enhancement of the drugs, having poor bioavailability, to minimize the dose of drug administered and to increase pharmacological action of drug. Vesicular systems have been realized as extensively helpful carrier systems in different scientific domains. Over the years, vesicular systems have been investigated as a crucial drug delivery system due to their flexibility to be tailored for diverse advantageous purposes. Despite of certain drawbacks (fusion, aggregation), pharmacosomes still play a crucial role in the selective targeting, and the controlled delivery of different drugs. Pharmacosomes is not only having higher entrapment efficiency but it can be preestablished, because drug itself in conjugation with lipids forms vesicles. The physicochemical stability of the pharmacosome depends on the physicochemical properties of the drug-lipid complex.Emulsomes are favourable approach for the formulation of drug compounds with poor aqueous solubility with varied oral bioavailability. The oral delivery of hydrophobic drugs can be made possible by emulsomes, which have been shown to substantially increase oral bioavailability.Recent trends are focused on formation of modified emulsomal solid or semisolid formulations as an alternative to the conventional liquid system.

\section{References}

1. Biju SS, Talegaonkar S, et al. (2006) Vesicular systems: An overview. Ind J Pharma Sci 68 (2): 141-153.

2. Doijad RC, Bhambere DS, Fakirappa MV, Deshmukh NV (2009) Formulation and characterization of vesicular drug delivery system for anti-HIV drug. Journal of Global Pharma Technology 1(1): 94-100. 
3. Ali Gamal Ahmed Al-kaf, et al. (2012) A review on pharmacosomes: an emerging novel vesicular drug delivery system. Universal Journal of Pharmaceutical Research 2(1): 21-24.

4. Arien A, Dupuy B (1997) Encapsulation of calcitonin in liposomes depends in the vesicle preparation method. J Microencapsul 14: 753760.

5. Singh SK, Rajera R, Nagpal K, Mishra DN (2011) Niosomes: A controlled and novel drug delivery system. Biol Pharm Bull 34: 945-953.

6. Vaizoglu O, Speiser PP (1986) Pharmacosomes: a novel drug delivery system, Acta Pharm Suec 23: 163-172.

7. Bommala Supraja, Saritha Mullangi (2019) An updated review on pharmacosomes, a vesicular drug delivery system. Journal of Drug Delivery and Therapeutics 9(1-s): 393-402.

8. Varshosaz J, Talari R, Mostafavi SA, Nokhodchi A (2008) Dissolution enhancement of gliclazide using in situ micronization by solvent change method, Pow Tech 187(3): 222-230.

9. Biju SS, Talegaonkar S, Mishra PR, Khar KR (2009) Vesicular System: An overveiw. Indian J. Pharm Sci 71(4): 421-427.

10. Kavitha D, Naga Sowjanya J, Shanker P (2010) Pharmacosomes: An emerging vesicular system. International Journal of Pharmaceutical Sciences Review and Research 5(3): 168-171.

11. P. H. Sharma, P. V. Powar, S. S. Sharma (2014) Pharmacosomes: A novel drug delivery system. The pharma innovations journal 3(10): 94-100.

12. Semalty A, Semalty M, Rawat BS, Singh D, Rawat MSM (2010) Development and evaluation of pharmacosomes of aceclofenac. Ind J of Pharm Sci 72(5): 576-581.

13. Seema MJ, Pournima M, Manisha K, Vilasrao K (2012) Novel vesicular system: an overview. J of Appl Pharm Sci 2(1): 193-202.

14. Sonam Ranga, Amit Kumar (2014) A Review on Pharmacosomes. Research and reviews: journal of pharmaceutics and nanotechnology 2(1): 7-12.

15. Thakur Sonam, Tibrewal Richa (2017) Pharmacosomes: An overview. International journal of pharmaceutical and biological science archieve 5(2): 1-7.

16. Solanki D, Patidar A, Kukde D (2016) Pharmacosomes-a review. International Journal of Pharmacy, Eng and Life Sci 12(3): 70-78.

17. Rewar S, Mirdha D, Rewar P (2014) A vital role of pharmacosome's on controlled and novel drug delivery, Asi J of Res in Biol and Pharm Scienc 2(4): 163-170.

18. Nagasamy VD, Kalyani K, Tulasi K, Swetha P, Shaik AA (2014) Pharmacosomes: a potential vesicular drug delivery system, Internat J of Pharm Scie and Drug Research 6(2): 90-94.

19. Shaheda Sultana SK, et al. (2014) An updated overview on pharmacosomes. Internationaljournal of universal pharmacy and bio sciences 3(3): 710-730.

20. Semalty A, Semalty M, Rawat B S, Singh D, Rawat MSM (2009) Pharmacosomes: The lipid based novel drug delivery system, Expert Opinion on Drug Delivery 6: 599-612.

21. Semalty A, Semalty M, Singh D, Rawat MSM (2009) "Development and physicochemical evaluation of pharmacosomes of diclofenac," Acta Pharmaceutica 59(3): 335-344.
22. Bhatt DA, Pethe AM (2010) Lipoidal technology-a promising drug delivery for poorly water soluble drugs.Int J Pharm Res Dev 2(7): 1-11.

23. Sunil Kamboj, Vipin Saini, et al. (2013) Vesicular drug delivery systems: A novel approach for drug targeting. International Journal of Drug Delivery 5(2): 121-130.

24. Kumar D, Sharma D, et al. (2012) Lipoidal soft hybrid biocarriers of supramolecular construction for drug delivery. IntScholar Res Network Pharm 2012: 1-14.

25. Gill V, Kumar MS, et al. (2011) Development of amphotericin B loaded modified emulsomes for visceral leishmaniasis: in vitro. Int J Recent Adv Pharm Res 2: 14-20.

26. Bhawandeep Gill, Jatinder Singh, et al. (2012) Emulsomes: An emerging vesicular drug delivery system. Asian Journal of Pharmaceutics 6: 87-94

27. Rajesh Kumar, Nirmala, et al. (2013) Emulsomes: An emerging vesicular drug delivery system. Journal of Drug Delivery \& Therapeutics 3(6): 133-142.

28.Gupta S, Vyas SP (2006) Development and characterization of Amphotericin B bearing emulsomes for passive and active macrophage targeting. J Drug Target 15: 206-217.

29. Gupta KS, Nappinnai M, Gupta VRM (2010) Formulation and evaluation of topical meloxicam niosomal gel. Int J Biopharm 1: 7-13.

30. Kulthe VV, Chaudhari PD (2011) Solubility enhancement of etoricoxib by solid dispersions prepared by spray drying technique. Int J Pharm Res 45: 248-258.

31. Solid Fat Nanoemulsions As Drug Delivery Vehicles-Patent 5576016.

32. Banerjee A, Roychoudhury J, Ali N (2008) Stearylamine-bearing cationic liposomes kill leishmania parasites through surface exposed negatively charged phosphatidylserine. J Antimicrob Chemother 61: 103-110.

33. Azeem A, Anwer MK, Talegaonkar S (2009) Niosomes in sustained and targeted drug delivery: Some recent advances. J Drug Target 17: 671689.

34. Vyas SP, Subhedar R, Jain S (2006) Development and characterization of emulsomes for sustained and targeted delivery of an antiviral agent to liver. J Pharm Pharmacol 58(3): 321-326.

35. Paliwal R, Paliwal SR, Mishra N, et al. (2009) Engineered chylomicron mimicking carrier emulsome for lymph targeted oral delivery of methotrexat. Int J Pharm 380(1-2): 181-188.

36. Sudhamani T, Priyadarisini N, Radhakrishna (2010) Proniosomes-A promising drug carriers. Int J PharmTech Research 2(2): 1446-1454.

37. Vilhemsen T, Eliasen H, Schaefer T (2005) Effect of a melt agglomeration process on agglomerates containing solid dispersions. Int J Pharma 303(1-2): 132-142.

38. Erion MD, Van Poelje PD, et al. (2005) Liver-targeted drug delivery using hepdirect prodrugs. J Pharmacol Exp Ther 312: 554-560.

39. Moghbel A, Zand Moghaddam A, et al. (2003) High performance liquid chromatography determination of methotrexate in plasma. Iran J Pharm Res 2: 149-152.

40. Sanchez-Brunete JA, Dea MA, , et al. (2004) Treatment of experimental visceral leishmaniasis with amphotericin $B$ in stable albumin microspheres. Antimicrob Agents Chemother 48: 3246-3252. 
CC (P) This work is licensed under Creative

To Submit Your Article Click Here: Submit Article

DOI: 10.32474 /GJAPM.2020.03.000166

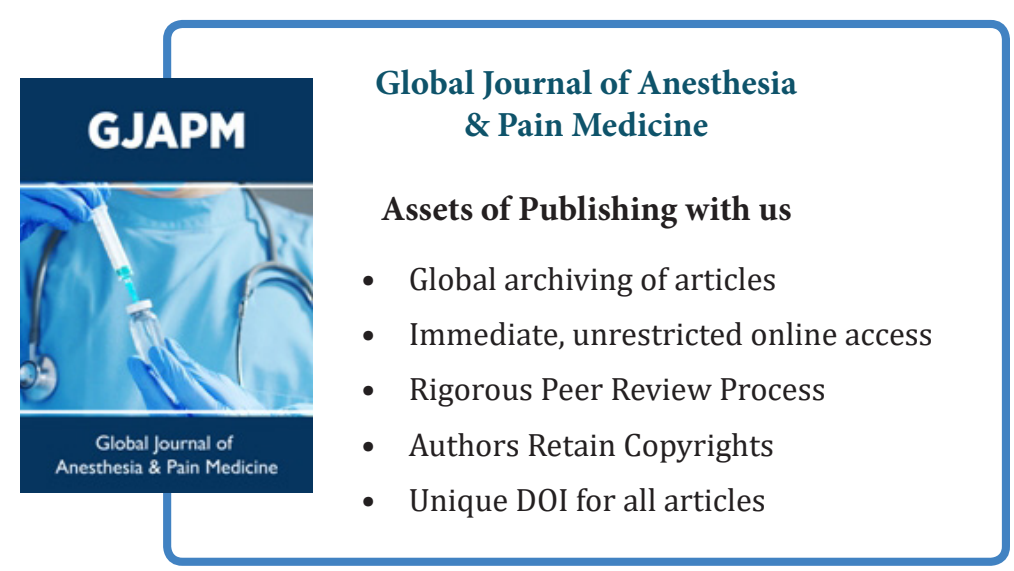

